\title{
Advances in the Diagnosis and Management of Differentiated Thyroid Cancer
}

\author{
a report by
}

Furio Pacini, ${ }^{1}$ Maria Grazia Castagna ${ }^{2}$ and Lucia Brilli²

\author{
1. Professor and Chairman; 2. Physician, Section of Endocrinology and Metabolism, \\ Department of Internal Medicine, Endocrinology and Metabolism and Biochemistry, University of Siena
}

DOI:10.17925/EE.2008.04.02.100

Thyroid cancer accounts for fewer than $1 \%$ of all human tumours and is much more prevalent among females than males. The incidence of thyroid cancer (mainly differentiated) is one of the most rapidly increasing among human cancers, at least in the US. ${ }^{1}$ This phenomenon is mainly due to an increase in the papillary histotype, which showed a 2.9-fold increase per year. The increase is attributable to better detection of small papillary carcinomas as a result of improved diagnostic accuracy (neck ultrasound and fine-needle aspiration cytology [FNAC]). It is a common experience in thyroid cancer referral centres that nearly $60-80 \%$ of thyroid carcinomas detected nowadays are micropapillary thyroid carcinomas (less than $1 \mathrm{~cm}$ in size) carrying an excellent long-term prognosis., , $3^{3}$ Despite an increasing incidence, the mortality from thyroid cancer in general and from papillary thyroid cancer in particular remained stable ( 0.5 deaths per 100,000 in both 1973 and 2002).1

In addition, in recent decades the clinical presentation of differentiated thyroid cancer (DTC) has been changing from advanced cases requiring intense treatment and surveillance to cancer detected by fortuitous neck ultrasonography requiring less aggressive treatment and follow-up. Diagnostic and treatment tools have also improved in recent years (sensitive assays for serum thyroglobulin measurement, neck ultrasound and recombinant human thyroid stimulating hormone

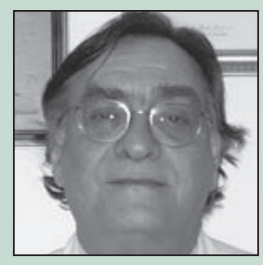

Furio Pacini is a Professor of Endocrinology and Chairman of the Section of Endocrinology and Metabolism at the University of Siena. He has been involved in both clinical practice and research in thyroidology and thyroid cancer in particular. Professor Pacini's research activity is characterised by hormonal, cellular and molecular approaches coupled with clinical observation, successfully bridging bench research and clinical practice, as testified in more than 150 publications.

E: pacini8@unisi.it

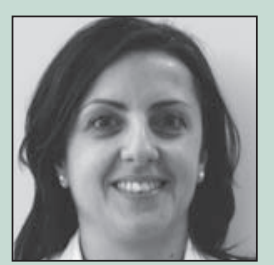

Maria Grazia Castagna is a Physician in the Department of Endocrinology at the University of Siena. She is a member of the European and Italian Thyroid Associations and the Italian Society of Endocrinology. She has authored or co-authored over 20 manuscripts and book chapters in the field of thyroid cancer, and is sub-investigator in several studies in clinical oncology.

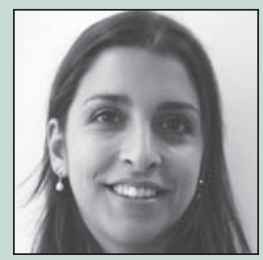

Lucia Brilli is a Physician in the Department of Endocrinology at the University of Siena. Her clinical and research interests focus on the diagnosis and treatment of thyroid diseases, in particular the management of patients with thyroid cancer; for this purpose she has participated as sub-investigator in several clinical studies using targeted therapies. She is a member of the European and Italian Thyroid Associations.
[rhTSH]), thus allowing for less invasive procedures for the patients. Altogether, these considerations dictate the need for applying the more effective and less expensive procedures able to guarantee the best management and the best quality of life for a cancer that, despite having an intrinsic low mortality, requires life-long follow-up.

\section{Diagnosis of Thyroid Cancer}

Thyroid cancer presents as a thyroid nodule detected by palpation and more frequently by neck ultrasound. While thyroid nodules are frequent (4-50\% depending on the diagnostic procedures and the age of the patient), thyroid cancer is rare (only $5 \%$ of all thyroid nodules). FNAC should be performed in any thyroid nodule greater than $1 \mathrm{~cm}$ and in those smaller than $1 \mathrm{~cm}$ if there is any clinical (history of head and neck irradiation, positive family history of thyroid cancer, suspicious features at palpation, presence of cervical adenopathy) or ultrasonographical suspicion of malignancy (hypoechogenicity, microcalcifications, absence of peripheral halo, irregular borders and regional lymphadenopathy). The results of FNAC are very sensitive for the differential diagnosis of benign and malignant nodules, although there are limitations: inadequate samples and follicular neoplasia. In the event of inadequate samples FNAC should be repeated while in the case of follicular neoplasia, with normal TSH and 'cold' appearance at thyroid scan, surgery should be considered. ${ }^{4-7}$ Considering that the prevalence of indeterminate FNAC is significant (10-25\%), several molecules involved in the carcinogenic process have been proposed as markers of thyroid malignancy, but none is recommended because of insufficient data. 8,9

\section{Initial Treatment}

The initial treatment for DTC is total or near-total thyroidectomy whenever the diagnosis is made before surgery, regardless of nodule size. Less extensive surgical procedures may be accepted in cases of unifocal DTC diagnosed at finally histology after surgery performed for benign thyroid disorders provided that the tumour is small, intrathyroidal and of favourable histological type (classic papillary or follicular variant of papillary or minimally invasive follicular). Surgery is usually followed by the administration of 131/ activities aimed at ablating any remnant thyroid tissue and eventually microscopic residual tumour. This procedure decreases the risk of locoregional recurrence and facilitates the long-term surveillance based on serum $\mathrm{Tg}$ measurement and diagnostic radioiodine whole body scan (WBS). In addition, the high activity of 131 | allows a highly sensitive post-therapeutic WBS to be obtained. Radioiodine ablation is recommended in high- and low-risk patients, while there is no indication in very low-risk patients (those with unifocal T1 tumours less than $1 \mathrm{~cm}$ in size, with favourable histology, no extrathyroidal extension and lymph node metastases). ${ }^{6}$ 
Traditionally, withdrawal of thyroid hormone has been used to optimise the trapping and retention of radioiodine by increasing endogenous TSH levels. The consequent state of hypothyroidism is unpleasant for most patients and sometimes results in marked morbidity and impairment of quality of life. ${ }^{10}$ Since 1995, rhTSH has been employed in clinical trials for post-surgical thyroid remnant ablation in DTC patients. A study published in 2001 demonstrated $100 \%$ ablation using ${ }^{131}$ activity determined by individual dosimetry (average dose of $110.3 \mathrm{mCi}$ ). ${ }^{11}$ Recently, a randomised, multicentre, controlled study ${ }^{12}$ designed to investigate whether preparation of patients with rhTSH while on levothyroxine suppressive (LT4) therapy was equivalent to preparation by LT4 withdrawal demonstrated comparable remnant ablation rates $(100 \%)$ in patients treated with a fixed dose of $100 \mathrm{mCi}$ of 131 I $(3,700 \mathrm{MBq})$ by either administering rhTSH or withholding thyroid hormone. rhTSH-prepared patients maintained a higher quality of life ${ }^{13}$ and received less radiation exposure to the body. ${ }^{14}$ Based on these results, the use of rhTSH for thyroid remnant ablation has been approved by the European Medicines Agency (EMEA) in February 2005 and by US Food and Drug Administration (FDA) in December 2007. More recently, another randomised prospective study employing rhTSH preparation for thyroid ablation found that $50 \mathrm{mCi}$ and $100 \mathrm{mCi}$ of 131 I were equally effective even in the presence of lymph node metastases. ${ }^{15}$ Based on this evidence, rhTSH may be considered to be the treatment of choice for post surgical thyroid ablation.

\section{Short-term Follow-up}

Six to 12 months after initial treatment, follow-up aims to ascertain whether the patient is free of disease, and is based on physical examination, neck ultrasound, serum Tg measurement and diagnostic 131| WBS.6,7 Currently, most of the patients (nearly $80 \%$ ) will be in complete remission as indicated by no clinical evidence of residual disease, unremarkable neck ultrasound and undetectable basal and stimulated serum $\mathrm{Tg}$ (in the absence of serum $\mathrm{Tg}$ antibodies).

A diagnostic 131। WBS does not add any clinical information in this setting and may be omitted. In association with a negative posttherapy WBS obtained at the time of thyroid remnant ablation, these findings are sufficient to classify the patient as free of disease, and the rate of subsequent recurrence in this group is very low $(<1.0 \%$ at 10 years). ${ }^{16,17}$ These patients may be shifted from suppressive to

\section{More recently, another randomised prospective study employing rhTSH preparation for thyroid ablation found that $50 \mathrm{mCi}$ and $100 \mathrm{mCi}$ of ${ }^{131}$ I were equally effective even in the presence of lymph node metastases.}

replacement LT4 therapy, with the goal of maintaining serum TSH level within the normal range. A significant proportion of patients defined as high-risk at the time of diagnosis may appear to be free of disease at their first follow-up after initial treatment. However, the risk of relapse in the long term may be significant; therefore, it is advisable to maintain these patients on suppressive doses of LT4 therapy (TSH around $0.1 \mu \mathrm{Ul} / \mathrm{ml}$ ) for a further three to five years. ${ }^{18}$

\section{Long-term Follow-up}

The subsequent follow-up of patients considered to be free of disease at the time of their first follow-up will consist of physical examination,

The subsequent follow-up of patients considered to be free of disease at the time of their first follow-up will consist of physical examination, basal serum Tg measurement on LT4 therapy and neck ultrasound once yearly.

basal serum Tg measurement on LT4 therapy and neck ultrasound once yearly. No other biochemical or morphological tests are indicated unless some new suspicion arises during evaluation.

The question of whether a second rhTSH-stimulated Tg test should be performed in disease-free patients is a matter of debate. Recent studies reported that this procedures has little clinical utility in patients who had no biochemical (undetectable serum Tg) or clinical (imaging) evidence of disease at the time of their first rhTSH$\mathrm{Tg}$. In this group, the second test confirmed complete remission in almost all patients. ${ }^{19,20}$

The small subgroup of patients with evidence of persistent disease after initial treatment may require further treatment. When the disease is limited to locoregional lymph nodes the option is between additional surgery or 131 I treatment (if there is 131। uptake in their metastases). Distant metastases are best treated by surgery whenever possible (resectable isolated localisation). When surgery is not feasible, radioiodine is the treatment of choice if the neoplastic tissue retained the expression of the sodium-iodide symporter (NIS) gene. Evidence has shown that distant metastases particularly in the lung are treated by radioiodine in about $40 \%$ of patients. ${ }^{20}$ After cumulative doses of $22 \mathrm{GBq}$ of $131 \mathrm{l}$, further treatment is usually ineffective. ${ }^{21}$

During the evaluation of metastatic patients, positron emission tomography with 18F-fluorodeoxyglucose (18FDG-PET) scanning is gaining attention as a diagnostic and prognostic tool.22 131/ WBSnegative and 18FDG-PET-positive patients indicate a group of patients with more aggressive and less differentiated disease carrying a worse prognosis with respect to 131 I-WBS-positive and 18 FDG-PET-negative patients, who have less aggressive disease and better prognosis.

\section{Targeted Therapy}

Diffuse metastatic disease refractory to radioiodine may be treated with systemic chemotherapy, but unfortunately the results are usually very poor and disappointing. ${ }^{6}$ Other currently available therapies are only palliative. For several years novel drugs developed to inhibit the function of specific oncoproteins (targeted therapies) have been intensively studied, aiming at shutting down the uncontrolled growth 
of neoplastic cells without harming normal cells. Papillary thyroid cancer is characterised by mutations leading to constitutive activation of multiple effectors that signal via the MAP kinase pathway. Molecules that block kinase activity at distal steps in the MAP kinase pathway are logical candidate drugs for papillary thyroid cancer.

TK inhibitors being tested against DTC in clinical trials include motesanib diphosphate, axitinib, gefitinib, sorafenib and sunitinib.

Several drugs inhibiting tyrosine kinase (TK) receptors have already been used successfully and some of them have enter clinical practice (i.e. imatinib in chronic myeloid leukaemia). TK inhibitors being tested against DTC in clinical trials include motesanib diphosphate, axitinib, gefitinib, sorafenib and sunitinib. None of these is specific for one oncogene protein, but they all target several TK receptors and proangiogenic growth factors. The results of phase II-III clinical trials conducted so far are promising.

Administration of motesanib diphosphate, an agent that targets vascular endothelial growth factor (VEGF), platelet-derived growth factor receptor (PDGFR), KIT and RET receptor tyrosine kinases (RTKS), in a single-arm multicentre phase II trial resulted in partial response (PR) and stable disease (SD) rates of 14 and 67\%, respectively, in 93 subjects with DTC and tolerable and manageable toxicities. ${ }^{23}$
Axitinib, an agent that targets all VEGF receptors, PDGFR- $\beta$ and KIT, was evaluated in a single-arm study of 60 subjects with advanced thyroid cancer and reported PR and SD rates in 13 patients (22\%) and in 30 patients (50\%), respectively. ${ }^{24}$

Gefitinib, a small-molecule inhibitor of the EGFR tyrosine kinase, was used to treat 27 patients with radioiodine-refractory, locally advanced or metastatic thyroid cancer of different histotypes, including papillary, follicular, medullary and Hurthle cell carcinoma. No objective responses were observed, although tumour reduction that did not meet the criteria for partial response was achieved in $32 \%$ of the patients and five patients with stable disease had a decrease in thyroglobulin to $<90 \%$ of baseline. In addition, stabilisation of the disease was obtained in 48,24 and $12 \%$ of the patients after three, six and 12 months of treatment, respectively. The authors interpreted these results as modest but still suggestive of a biological effect of the drug. ${ }^{25}$

The administration of sorafenib in 30 patients with metastatic, iodine-refractory thyroid carcinoma determined a PR in seven patients (23\%), a SD in 16 patients (53\%) and showed an overall clinical benefit rate (partial response plus stable disease) of $77 \%$, a median progression-free survival of 79 weeks and an overall acceptable safety profile. ${ }^{26}$ Sunitinib is a inhibitor of VEGFR-1, VEGFR-2, foetal liver tyrosine kinase receptor 3 (FLT3), KIT and PDGFR in both biochemical and cellular assays, but the response rate in clinical trials is still being evaluated.

\section{Conclusion}

The preliminary results of these trials are promising and indicate that targeted therapy might become the first-line treatment of metastatic refractory thyroid cancer patients in the near future.
1. Davies $L$, Welch $H G$, Increasing incidence of thyroid cancer in the US, 1973-2002, JAMA, 2006;295:2164-7.

2. Leenhardt L, Bernier MO, Boin-Pineau MH, et al., Advances in diagnostic practices affect thyroid cancer incidence in France, Eur J Endocrinol, 2004;150:133-9.

3. Levi F, Randimbison L, Te VC, et al. Thyroid cancer in Vaud, Switzerland: an update, Thyroid, 2002;12:163-8

4. Pacini F, Burroni L, Ciuoli C, Di Cairano G, Guarino E, Management of thyroid nodules: a clinicophatological evidence-based approac, Eur I Nucl Med Mol Imaging, 2004;31:1443-49.

5. Cap J, Ryska A, Rehorkova P, et al., Sensitivity and specificity of the fine needle aspiration biopsy of the thyroid: clinical point of view, Clin Endocrinol, 1999;51:509-15.

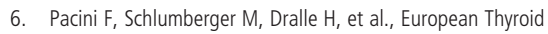
Cancer Taskforce.. European consensus for the management of patients with differentiated thyroid carcinoma of the follicular epithelium, Eur J Endocrinol, 2006;154:787-803.

7. Cooper DS, Doherty GM, Haugen BR, et al., The American Thyroid Association Guidelines Taskforce. Management guidelines for patients with thyroid nodules and differentiated thyroid cancer, Thyroid, 2006;16:109-42.

8. Shibru D, Chung KW, Kebebew E, Recent developments in the clinical application of thyroid cancer biomarkers, Curr Opin Oncol, 2008;20:13-18.

9. Griffith OL, Melck A, Jones SJ, Wiseman SM, Meta-analysis and meta-review of thyroid cancer gene expression profiling studies identifies important diagnostic biomarkers, I Clin Oncol, 2006;24:5043-51

10. Schroeder PR, Haugen BR, Pacini F, et al., A comparison of short-term changes in health-related quality of life in thyroid carcinoma patients undergoing diagnostic evaluation with recombinant human thyrotropin compared with thyroid hormone withdrawal, J Clin Endocrinol Metab, 2006;91:
878-84.

11. Robbins RJ, Tuttle RM, Sonenberg M, et al., Radioiodine ablation of thyroid remnants after preparation with recombinant human thyrotropin, Thyroid, 2001;11:865-9.

12. Pacini F, Ladenson PW, Schlumberger M, et al. Radioiodine ablation of thyroid remnants after preparation with recombinant human thyrotropin in differentiated thyroid carcinoma: results of an international, randomized, controlled study, J Clin Endocrinol, 2006:91:926-32

13. Schroeder PR, Haugen BR, Pacini F, et al., A comparison of short-term changes in health-related quality of life in thyroid carcinoma patients undergoing diagnostic evaluation with recombinant human thyrotropin compared with thyroid hormone withdrawal, J Clin Endocrinol Metab, 2006;91: 878-84.

14. Hänscheid H, Lassmann M, Luster M, Thomas SR, et al., Reiners C.lodine biokinetics and dosimetry in radioiodine therapy of thyroid cancer: procedures and results of a prospective international controlled study of ablation after rhTSH or hormone withdrawal, J Nucl Med, 2006;47:648-54.

15. Pilli T, Brianzoni E, Capoccetti F, et al., A comparison of 1850 (50mCi) and $3700 \mathrm{MBq}(100 \mathrm{mCi}) 131$-iodine administered doses for recombinant thyrotropin-stimulated postoperative thyroid remnant ablation in differentiated thyroid cancer, J Clin Endocrinol Metab, 2007:92:3542-6.

16. Pacini F, Capezzone M, Elisei R, et al., Diagnostic 131-iodine whole-body scan may be avoided in thyroid cancer patients who have undetectable stimulated serum Tg levels after initial treatment, I Clin Endocrinol Metab, 2002;87:1499-501.

17. Cailleux AF, Baudin E, Travagli JP, et al.,Is diagnostic iodine-131 scanning useful after total thyroid ablation for differentiated thyroid cancer? I Clin Endocrinol Metab, 2000;85:175-8.

18. Biondi B, Filetti S, Schlumberger M, Thyroid-hormone therapy and thyroid cancer: a reassessment, Nat Clin Pract Endocrinol Metab, 2005;1:32-40

19. Castagna MG, Brilli L, Pilli T, et al., Limited value of repeat recombinant human thyrotropin (rhTSH)-stimulated thyroglobulin testing in differentiated thyroid carcinoma patients with previous negative rhTSH-stimulated thyroglobulin and undetectable basal serum thyroglobulin levels, J Clin Endocrinol Metab, 2008;9376-81.

20. Kloos RT, Mazzaferri EL, A single recombinant human thyrotropin-stimulated serum thyroglobulin measurement predicts differentiated thyroid carcinoma metastases three to five years later, I Clin Endocrinol Metab, 2005;90:5047-57.

21. Durante C, Haddy N, Baudin E, et al., Long-term outcome of 444 patients with distant metastases from papillary and follicular thyroid carcinoma: benefits and limits of radioiodine therapy, J Clin Endocrinol Metab, 2006;91:2892-9.

22. Robbins RJ, Wan Q, Grewal RK, et al., Realtime prognosis for metastatic thyroid carcinoma based on 32-[18F]fluoro-2-deoxy-D-glucose-positron emission tomography scanning, J Clin Endocrinol Metab, 2006;91:498-505.

23. Sherman SI, Wirth CJ, Droz JP, et al.; for the Motesanib Thyroid Cancer Study Group, Motesanib Diphosphate in Progressive Differentiated Thyroid Cancer, N Engl I Med, 2008;359:31-42

24. Cohen $E E, A$ phase II study of axinitib in patients with advanced thyroid cancers. Abstract 6008 presented at the 43rd Annual Meeting of the American Society of Clinical Oncology (ASCO), 2007.

25. Pennell NA, Daniels GH, Haddad RI, et al., A phase II study of gefitinib in patients with advanced thyroid cancer, Thyroid, 2008; 18:317-23.

26. Gupta-Abramson V, Troxel AB, Nellore A, et al., Phase II Trial of Sorafenib in Advanced Thyroid Cancer, J Clin Oncol, 2008:26:4714-19. 